Research Article

\title{
Impact of Community-Led Total Sanitation and Hygiene on Prevalence of Diarrheal Disease and Associated Factors among Under-Five Children: A Comparative Cross-Sectional Study in Selected Woredas of Gamo Gofa Zone, Southern Ethiopia
}

\author{
Agune Ashole Alto $(\mathbb{D}$, Wanzahun Godana $(\mathbb{D}$, and Genet Gedamu \\ Department of Public Health, Arba Minch University, Arba Minch, Ethiopia \\ Correspondence should be addressed to Agune Ashole Alto; ozilagune@gmail.com
}

Received 1 May 2019; Revised 10 December 2019; Accepted 31 January 2020; Published 24 February 2020

Academic Editor: Carol J. Burns

Copyright (c) 2020 Agune Ashole Alto et al. This is an open access article distributed under the Creative Commons Attribution License, which permits unrestricted use, distribution, and reproduction in any medium, provided the original work is properly cited.

\begin{abstract}
Background. Diarrheal diseases are still one of the major causes of morbidity in under-five children in sub-Saharan Africa. In Ethiopia, diarrhea is responsible for $9 \%$ of all deaths and is the major cause of under-five mortality. Objective. To assess the impact of community-led total sanitation and hygiene on the prevalence of diarrheal disease and factors associated among under-five children in Gamo Gofa Zone. Methods. Community-based comparative cross-sectional study design was used to compare the impact of community-led total sanitation and hygiene intervention on under-five diarrheal disease. Multistage sampling method was employed. The data were collected by using pretested structured questionnaires. Data quality was ensured by daily supervision completeness and consistency. The data were coded, entered, and cleaned by using Epi Info version 7 and were analyzed by using SPSS version 20. Bivariate and multivariable analyses were carried out by using binary logistic regression. Significance was declared by using $p$ value of $<0.05$ and AOR with $95 \%$ confidence intervals. Results. The response rate of this study was $93.3 \%$. The overall diarrhea prevalence was $27.5 \%(\mathrm{CI}=(24.06,30.97))$ which was $18.9 \%(\mathrm{CI}=(14.94,23.2))$ in implemented and $36.2 \%$. $(\mathrm{CI}=(30.41,41.59))$ in nonimplemented woredas. Children whose age was between 12 and 23 months $(\mathrm{AOR}=1.6)$ and greater than 24 months $(\mathrm{AOR}=5)$, availability of handwashing facilities $(\mathrm{AOR}=4)$, disposal of waste in open field $(\mathrm{AOR}=9.7)$, unimproved source of drinking water $(\mathrm{AOR}=6.5)$, using only water for handwashing $(\mathrm{AOR}=6)$, children who started complementary feeding less than 6 months $(\mathrm{AOR}=5.6)$ and greater than 6 months $(\mathrm{AOR}=5.2)$, and utensils used to feed children such as bottle $(\mathrm{AOR}=3.9)$ were the factors positively associated with diarrhea. Conclusion. The overall prevalence of under-five diarrhea was $27.5 \%$. The prevalence was low in CLTSH woredas as compared with non-CLTSH woredas. The study showed that handwashing facility, using only water for handwashing, open refuse disposal, and unimproved source of drinking water among under-five had a statistically significant association with diarrhea occurrence in CLTSH nonimplemented areas. Integrated efforts are needed from the Ministry of Health together with the WASH Project in improving drinking water, handwashing facilities, and solid waste disposal practices.
\end{abstract}

\section{Background}

Community-led total sanitation (CLTS) is an approach which is considered to be effective in triggering and mobilizing the societies at the community level. CLTS incentives support toilet construction for individual families and alternatively a top-down approach [1]. Instead, through processes of concentrated facilitation and mobilization and powerful emotions such as disgust and shame, the whole community is encouraged to analyze the problems associated with open field defecation in order to make free of open defecation communities [2].

CLTS was started in Bangladesh in 1999. It has spread speedily across Asia, Latin America, and Africa and is now 
being implemented in over 20 countries in the world [2]. In recent years, new novelties such as community-led total sanitation and hygiene have concerned attention. CLTSH has run to thousands of low-cost toilets springing up all around the rural community of Asia and Africa [2, 3].

In Ethiopia, it was implemented in 2011 and launched after two training events were organized and conducted in Arba Minch and Hawassa towns by VITA Ethiopia and Plan International Ethiopia, respectively. After these events, implementation of the approach led to visible and outstanding results in the direction of improving the hygiene and sanitation condition in the country. Based on this observation, a number of nongovernmental organizations started to implement the CLTSH approach in different parts of the country $[2,4]$. The Federal Ministry of Health (F$\mathrm{MoH}$ ) organized National Hygiene and Sanitation Task Force to reinforce the efforts being made to address hygiene and sanitation concerns in the country and to maximize the effectiveness and efficiency of CLTSH approach. And also, technical working group was organized in the same year by the National Hygiene and Sanitation Task Force to harmonizing the implementation of CLTSH by different areas of the continents. National CLTSH Training Manual, CLTSH Implementation Guideline, and ODF Status Verification Protocol were developed by the Ethiopian Ministry of Health and they were used to monitor, train, and verify societies [4].

Diarrhea is recurrent loose or watery bowel movements that depart from a child's normal pattern [5]. It is a very public pediatric concern and causes about 1.5 million deaths/year globally. Worldwide, it accounts for about $9 \%$ of hospitalizations among under-five children [6]. According to the WHO and UNICEF, internationally there are about 2 billion cases of diarrheal disease each year and 1.9 million (18\%) children under five years of age which means five hundred children die every day from diarrhea, frequently in developing countries. Of all these child deaths from diarrhea, $78 \%$ occur in the African continent [6]. In sub-Saharan Africa, diarrhea kills younger children more than measles, malaria, and AIDS. Episodes of acute diarrhea experience three times yearly in each under-five children [7].

Furthermore, improvement of water sanitation and hygiene promotion approaches is still required to reduce the burden of diarrheal diseases in children in Ethiopia in general and SNNPR in particular specifically in Gamo Gofa Zone. In this regard, there are studies carried out to identify factors associated with diarrheal diseases, but there are some research-based empirical evidences regarding sanitation and hygiene promotion approach effectiveness in CLTSH implementation in the country. Even if the implementation of CLTSH was started in some areas of Ethiopia, the valuation of CLTSH approach on the control of diarrheal disease was not assessed, particularly in the study area. Hence, this study was attempted to compare factors of diarrheal diseases among children in CLTSH approach implemented and nonimplemented areas. So, the objective of this study was to compare the prevalence of diarrheal disease among children in CLTSH implemented and nonimplemented woredas in Gamo Gofa Zone, Southern Ethiopia.

\section{Methods}

2.1. Study Area and Period. The study was conducted from March 1-March 30, 2018, in two selected CLTSH implemented and nonimplemented woredas of Gamo Gofa Zone. The Gamo Gofa Zone was administratively divided into fifteen rural and two urban woredas. CLTSH implementation was started in Gamo Gofa Zone five years before the study. Out of the woredas, there were five CLTSH implemented and five nonimplemented woredas. Uba Debretsehay (UDT) woreda from CLTSH implemented areas and Kamba woreda from CLTSH nonimplemented areas were selected woredas. Uba Debretsehay and Kamba were located $185 \mathrm{~km}$ and $100 \mathrm{~km}$ west of Arba Minch (the capital city of Gamo Gofa), respectively. The CLTSH implemented woredas have 19 and nonimplemented woredas have 38 rural kebeles. The total population of the woreda was about 90145 in Uba Debretsehay woreda (UDT) and 202279 in Kamba woreda whereas $51 \%$ were males in both areas. 14072 children less than five years of age in the years 2017/18 were in implemented woredas and 31576 children in nonimplemented woredas.

2.2. Study Design. A community-based comparative crosssectional study design was used.

\subsection{Population}

2.3.1. Source Population. All mothers or caretakers had at least one under-five children in selected CLTSH implemented and nonimplemented woredas.

2.3.2. Study Population. All mothers or caretakers had at least one under-five children living in the selected kebeles.

2.3.3. Inclusion Criteria. Mothers or immediate caretakers who had at least one under-five child and lived in the study area for about 6 months were included in the study.

2.3.4. Exclusion Criteria. Mothers or immediate caretakers who were critically ill were excluded from the study.

\subsection{Sample Size and Sampling Procedure}

2.4.1. Sample Size Determination. Sample size was calculated with double population proportion formula by using Epi Info 7 by using 1:1 ratio and $80 \%$ power. In the sample size calculation, diarrheal disease prevalence from other studies was considered and assumed as exposure (nonimplemented) which is from Dangla woreda, Northern Ethiopia [8], and $p_{2}$ was the outcome of nonexposure (implemented) which is from Kersa District, Jimma Zone [9] (see Table 1).

The sample size was obtained from objective 1 where the prevalence of under-five was 310. It was multiplied by 2 for design effect and $10 \%$ for nonresponse. The total sample size 
TABLE 1: Sample size determination of prevalence of diarrhea morbidity in CLTSH implemented and nonimplemented areas in Gamo Gofa Zone, 2018.

\begin{tabular}{|c|c|c|c|c|c|c|c|c|c|c|c|}
\hline $\begin{array}{l}\text { S. } \\
\text { no. }\end{array}$ & Main factors & CI & Power & Ratio & $\begin{array}{l}\text { Outcome in } \\
\text { unexposed } \\
\text { group }\left(p_{1}\right)\end{array}$ & $\begin{array}{l}\text { Outcome in } \\
\text { exposed }\left(p_{2}\right)\end{array}$ & OR & $\begin{array}{l}\text { Implemented } \\
\text { area }\end{array}$ & $\begin{array}{l}\text { Nonimplemented } \\
\text { area }\end{array}$ & Total & Reference \\
\hline 1 & \multicolumn{11}{|c|}{ Objective 1} \\
\hline 1.1 & Prevalence & $95 \%$ & $80 \%$ & $1: 1$ & 9.9 & 22.22 & 2.59 & 155 & 155 & 310 & {$[8,9]$} \\
\hline 2 & \multicolumn{11}{|c|}{ Objective 2} \\
\hline 2.1 & Water shortage & $95 \%$ & $80 \%$ & $1: 1$ & 3.1 & 12.6 & 4.5 & 145 & 145 & 290 & [8] \\
\hline 2.2 & $\begin{array}{l}\text { Handwashing } \\
\text { practice }\end{array}$ & $95 \%$ & $80 \%$ & $1: 1$ & 28.8 & 54.4 & 2.95 & 65 & 65 & 130 & {$[10]$} \\
\hline
\end{tabular}

was 682, i.e., 341 from CLTSH implemented and 341 from CLTSH nonimplemented areas.

2.4.2. Sampling Procedure. Multistage sampling method was employed. Out of 15 rural woredas, two woredas (one CLTSH implemented and one nonimplemented) were selected by lottery method from those five CLTSH implemented and five CLTSH nonimplemented areas. Then, from implemented woreda (19 kebeles) five kebeles and from nonimplemented woreda (38 kebeles) ten kebeles were randomly selected, and a systematic sampling method was used to select households from each kebele (see Figure 1).

\subsection{Study Variable}

2.5.1. Dependent Variable. The occurrence of acute diarrhea was the dependent variable.

2.6. Independent Variables. The following independent variables were used in this study:

(i) Sociodemographic or personal variables

(ii) Sanitation and hygiene-related variables

(iii) Water-related variables

(iv) Behavioral variables

(v) Child-related variables

\subsection{Operational Definitions}

(i) Diarrhea: diarrhea is defined as having three or more loose or watery stool in a $24 \mathrm{~h}$ period in the household during or within the two-week period prior to the survey, as reported by the mother/ caretaker of the child [11].

(ii) Index child: it refers to a child that was included in the study from a household; if there are many children in the home, the child with diarrhea is included in the study. If there is no child with diarrhea, one of the children will be randomly selected and included in the study [12].

(iii) Improved water sources: improved drinking water sources are defined as follows: piped water into dwelling, piped water into yard/plot, public tap/ standpipes, tube well/boreholes, protected dug wells, protected springs (normally part of a spring supply), and bottled water, if the secondary source used by the household for cooking and personal hygiene is improved [13]

(iv) Improved sanitation: an improved sanitation facility is defined as one that hygienically separates human excreta from human contact [13].

(v) Proper latrine utilization: households having functional latrines and no feces in the compound, observable fresh feces through the hole, and the footpath to the latrine should not be covered with grasses.

(vi) Proper handwashing facility: household having functional handwashing facility with water in the container and dampness under the container [13].

(vii) Good handwashing practice: washing both hands with soap or ash after using the toilet and cleaning the baby's bottom and washing hands before feeding children and before handling food practices at least three times out of five critical times of handwashing practice [14].

(viii) Proper refuse disposals: a way of disposal refuses which included burning, burying in a pit or storing in a container and disposing in designed site [13].

(ix) Community-led total sanitation (CLTSH): CLTSH is an approach used to improve sanitation and hygiene practices in a community ending the practice of open defecation. The sequence of steps and activities in CLTSH is as follows:

(1) Pretriggering: selecting a community, conducting baseline survey, and understanding administrative preparations before the appraisal

(2) Triggering: introduction and rapport building, participatory analysis, ignition movement, and action planning by the community

(3) Posttriggering: construction of latrines by the community, sustaining and monitoring the ODF status, external encouragement to the community, and verifying and certifying the ODF status

It can also include the following additional criteria [15]:

(i) Household latrines are hygienic, provide the safe containment of feces, offer privacy, and have a lid on 

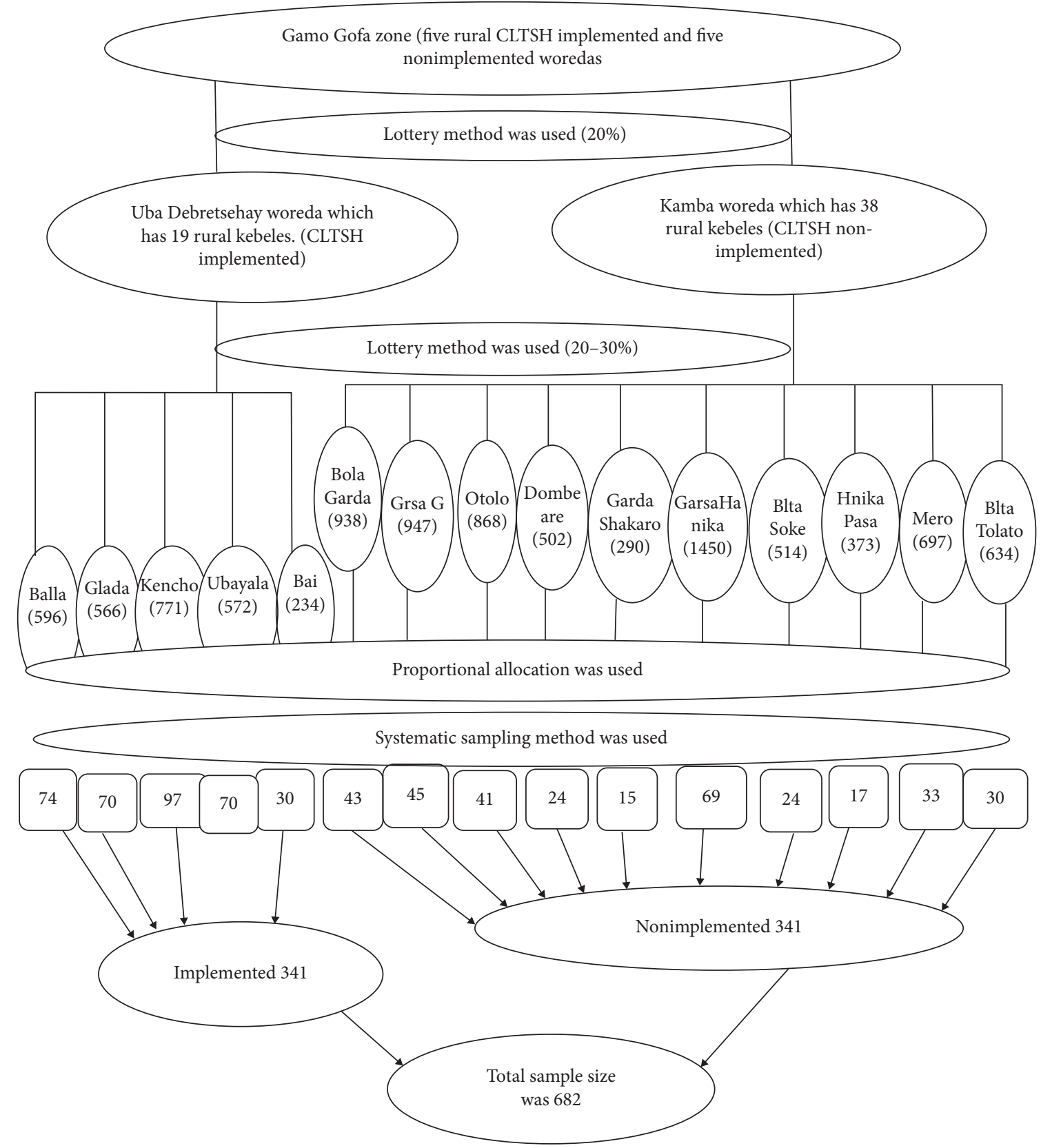

FIGURE 1: Schematic representation of sampling procedure on prevalence and factors associated with diarrhea among under-five in CLTSH implemented and nonimplemented areas in Gamo Gofa Zone, 2018.

the defecation hole or a water seal and a roof to protect the user

(ii) All household members and all members of the community use these toilets

(iii) A handwashing facility is nearby with water, soap, or ash and is used regularly

Even more stringent criteria which may be added to achieve "ODF status" for a community might include [15]

(i) Safe drinking water and storage

(1) Food hygiene

(2) Greywater disposal
(3) Solid waste management

(4) Provision of toilets at schools and markets and for passers-by [15]

2.8. Data Collection Procedure (Instrument and Personnel). The data collection tools were adapted from EDHS 2016 and other published literature studies $[8,16]$. It was initially prepared in English. The English version was translated to the Amharic language and back-translated to English by language experts to ensure consistency. The data were collected by using pretested structured questionnaires. Ten health professionals who have prior experience in data 
collection collected the data. Two public health officers from the different woreda health facilities supervised the collection. All data collectors and supervisors were trained for one day and performed practical exercises to be familiar with the questionnaire.

2.9. Data Quality Management. Before data collection, pretested structured data collection instrument was used. Pretest was done on 5\% of the sample in Zala woreda which is one of the unselected woredas to ensure clarity, wordings, logical sequence, and skip patterns of the questions. Based on the pretest, the time needed to complete an interview and the total number of days needed for data collection were estimated. During data collection, data collectors and data clerks were trained and closely supervised. Coding was developed and double entry was done to ensure data quality.

2.10. Data Processing and Analysis. The data were coded, cleaned, and entered by Epi Info version 7 and exported to Statistical Package for Social Science (SPSS) version 20.0 for analysis. Data were presented by using graphs, charts, tables, and proportion. Bivariate analysis was performed and explanatory variables with $p$ value $\leq 0.2$ and significant in other studies were entered into the final regression model. Multivariable logistic regression analysis was performed to estimate the association between outcome and explanatory variables. A $p$ value $\leq 0.05$ was considered statistically significant in this study. An effort was made to assess whether the necessary assumptions for the application of multivariable logistic regression are fulfilled. In this regard, Hosmer and Lemeshow's goodness-of-fit test with large $p$ value $(p>0.05)$ was checked to see good fitness. Multicollinearity and confounding effect were checked by using standard error, and variables with tolerance $<0.1$ and variance inflation rate more than $10 \%$ were excluded. Only variable with $p$ value $\leq 0.05$ was reserved in the final model. Finally, odds ratio along with $95 \%$ confidence interval (CI) were used to assess the association between explanatory variables and diarrheal prevalence.

\section{Results}

3.1. Sociodemographic Characteristics. A total of 636 participants (318 from CLTSH implemented and 318 from nonimplemented) participated in the study with the response rate of $93.3 \%$. The majority of index children (125, $39.3 \%$ ) in CLTSH implemented woreda were aged greater than 24 months and 129 (32.4\%) in nonimplemented were aged 6-11 months (see Figure 2).

Among the respondents, 285 (89.6\%) in CLTSH implemented woredas and $230(72.3 \%)$ in CLTSH nonimplemented woredas were mothers. The mean age was $29.6 \pm 7.6$ and $28.6 \pm 6.2$ in CLTSH implemented and nonimplemented woredas, respectively. The family size of the total respondents in the study area ranged from 2 to 14 people with a mean of $5.1 \pm 2.4$ in CLTSH implemented and from 2 to 13 people with a mean of $5.5 \pm 2.6$ in CLTSH nonimplemented woredas. Most of the mothers in the household (198 (62.3\%) among implemented and 189 (59.4\%) among nonimplemented) were of Gofa ethnicity and more than half in both implemented and nonimplemented were protestant (see Table 2).

3.2. Environmental Characteristics. The households having latrine were $75.5 \%$ in CLTSH implemented and $71.4 \%$ in nonimplemented woredas, respectively. Out of 467 latrine facilities, only 111 (46.2\%) of households in CLTSH implemented woredas and 94 (41.4\%) households in CLTSH nonimplemented woredas have handwashing facilities and most of the others did not have handwashing facilities. From a total of 636 households whose responses were obtained, 27 (8.5\%) in the CLTSH implemented and 43 (13.5\%) in the CLTSH nonimplemented woredas explained that they disposed wastes in open field in the compound. Regarding the source of drinking water, $240(75.5 \%)$ in CLTSH implemented and $212(66.7 \%)$ in nonimplemented woredas use drinking water from improved sources. The majority of the respondents in CLTSH implemented (254 (79.9\%)) and nonimplemented $(175(55 \%))$ woredas use jerry can as their main storage for drinking water. Out of 636 storage containers, $202(63.5 \%)$ in implemented and $240(75.5 \%)$ in nonimplemented households had no cover (see Table 3).

3.3. Behavioral Characteristics. The majority of the respondents in CLTSH implemented (286 (89.9\%)) and nonimplemented $(224(70.4 \%))$ woredas were washing their hands with soap/ash. Similarly, 280 (88\%) in implemented and $231(72.6 \%)$ in nonimplemented woredas wash their hands at critical times. 161 (50.6\%) in CLTSH implemented and $156(49.1 \%)$ in CLTSH nonimplemented woredas started complementary feeding for their children at six months, and among the respondents, 293 (92.1\%) in CLTSH implemented and $261(82.1 \%)$ nonimplemented woredas receive Rota vaccine (see Table 4).

3.4. Prevalence of Diarrhea. A total of 636 respondents, 318 households in CLTSH implemented and 318 households in CLTSH nonimplemented woredas, participated in the study. The finding of this study showed that the two-week period magnitude of diarrhea among under-five children was $27.5 \%$ $(24.06,30.97)$ which was $18.9 \%(14.94,23.20)$ in CLTSH implemented and $36.2 \%(30.41,41.59)$ in nonimplemented woredas. The occurrence of diarrhea has a significant difference between the two CLTSH implemented and nonimplemented woredas with $F$ value of 24.69 and $p$ value of 0.001 (see Figure 3).

\subsection{Bivariate Regression Analysis Results}

3.5.1. Sociodemographic Factors Related to Diarrhea. The unadjusted odds ratio results revealed significant differences in the effects of different variables on under-five diarrhea. However, because no controls were introduced at this level, assessing the independent predictors of childhood diarrhea was impossible. From all sociodemographic variables, only 


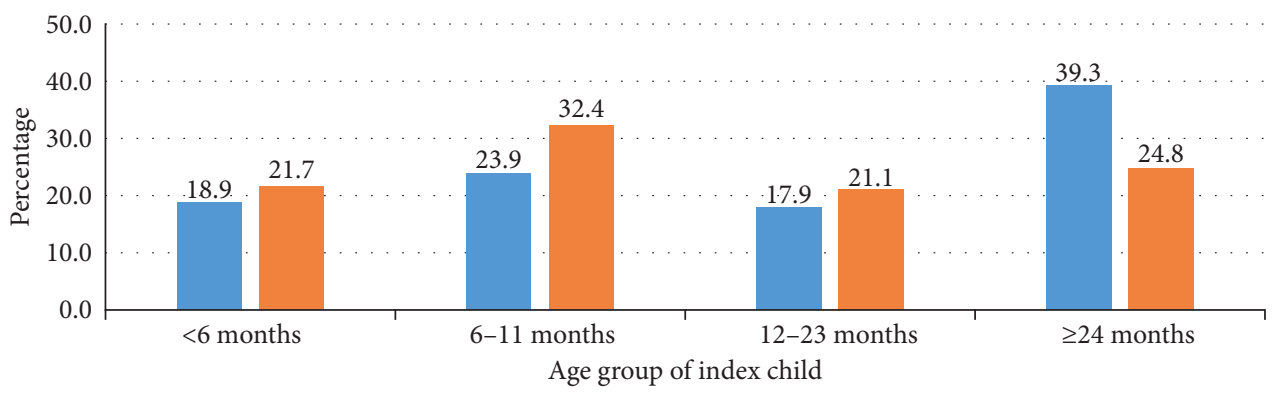

- CLTSH implemented

- CLTSH nonimplemented

Figure 2: Bar chart shows the prevalence of age of index child among under-five in CLTSH implemented and nonimplemented areas in Gamo Gofa Zone, March 2018.

TABle 2: The sociodemographic characteristics in selected CLTSH implemented and nonimplemented woreda in Gamo Gofa Zone, 2018.

\begin{tabular}{|c|c|c|c|c|}
\hline Variables & Characteristics & $\begin{array}{c}\text { CLTSH implemented (318) } \\
n(\%)\end{array}$ & $\begin{array}{l}\text { CLTSH nonimplemented (318) } \\
n(\%)\end{array}$ & $\begin{array}{c}\text { Total } \\
N(\%)\end{array}$ \\
\hline \multirow{2}{*}{ Caretaker of index child } & Mother & $285(89.6 \%)$ & $230(72.3 \%)$ & $515(11 \%)$ \\
\hline & Caregiver & $33(10.4 \%)$ & $88(27.7 \%)$ & $121(19 \%)$ \\
\hline \multirow{3}{*}{ Age of caretaker } & $15-24$ & $100(3.5 \%)$ & $84(26.4 \%)$ & $184(28.9 \%)$ \\
\hline & $25-34$ & $108(33.9 \%)$ & $173(54.4 \%)$ & $281(44.2 \%)$ \\
\hline & $\geq 35$ & $110(34.6 \%)$ & $61(19.2 \%)$ & $171(28.9 \%)$ \\
\hline \multirow{5}{*}{ Marital status } & Married & $238(74.8 \%)$ & $191(60 \%)$ & $429(67.5 \%)$ \\
\hline & Single & $22(6.9 \%)$ & $37(11.6 \%)$ & $59(9.3 \%)$ \\
\hline & Widowed & $24(7.6 \%)$ & $31(9.8 \%)$ & $55(8.7 \%)$ \\
\hline & Divorced & $22(6.9 \%)$ & $40(12.6 \%)$ & $62(9.7 \%)$ \\
\hline & Separated & $12(3.8 \%)$ & $19(6 \%)$ & $31(4.9 \%)$ \\
\hline \multirow{3}{*}{ Monthly income } & $<1000$ ETB & $103(32.4 \%)$ & $57(17.9 \%)$ & $160(25.2 \%)$ \\
\hline & $1000-2000$ ЕTВ & $29(9.1 \%)$ & $75(23.6 \%)$ & $104(16.4 \%)$ \\
\hline & $\geq 2000$ ETB & $186(58.5 \%)$ & $186(58.5 \%)$ & $372(58.5 \%)$ \\
\hline \multirow{2}{*}{ Number of $<5$ children } & One & $276(86.8 \%)$ & $276(86.8 \%)$ & $552(86.8 \%)$ \\
\hline & More than one & $42(13.2 \%)$ & $42(13.2 \%)$ & $84(13.2 \%)$ \\
\hline \multirow{2}{*}{ Sex of the child } & Male & $168(52.8 \%)$ & $173(54.4 \%)$ & 341 (53.6\%) \\
\hline & Female & $150(47.2 \%)$ & $145(45.6 \%)$ & $295(46.4 \%)$ \\
\hline
\end{tabular}

age fulfilled the criteria for multivariable analysis (see Table 5).

3.5.2. Environmental Factors Related to Diarrhea. From environmental factors, the variables which are the candidates for multivariable analysis were latrine availability, improper refuse disposal, absence/presence of handwashing facilities, and unimproved source of drinking (see Table 6).

3.5.3. Behavioral Factors Related to Diarrhea. According to behavioral factors, the candidates for multivariable analysis were washing hands without soap/ash, materials used to feed child such as hand and bottle, using plastic bucket, iron bucket, and pot for water storage, child started supplementary feeding below six months and above six months, and Rota vaccine (see Table 7).

3.6. Multivariable Regression Analysis Results. The logistic regression technique was used to assess the relative effect of the explanatory variable on the outcome variable. To avoid an excessive number of variables and unstable estimates in the subsequent model, only variables with a $p$ value of less than 0.20 were kept in the subsequent analyses. The multivariable analysis result showed that from the total thirteen variables, seven variables were found to have significant independent association with diarrhea.

Keeping other factors constant, children whose age was between 12 and 23 months and greater than 24 months were $1.6(\mathrm{AOR}=1.58 ; 95 \% \mathrm{CI}:(1.5-8.56))$ and $5(\mathrm{AOR}=5.23 ; 95 \%$ CI: (2.12-12.8)) times more likely to have diarrhea as compared to children whose age was less than six months, respectively. There is no difference in age in CLTSH implemented and nonimplemented areas with $F$ value of 2.218 and $p$ value of 0.09 .

Regarding environmental factors, holding other factors constant, children whose family did not have handwashing facilities had 4 times higher chance $(\mathrm{AOR}=4.03$; 95\% CI: (1.93-8.42)) of diarrhea occurrence as compared to children of the family who have handwashing facility. There is difference in handwashing facilities in CLTSH implemented 
TABLE 3: Environmental characteristics in selected CLTSH implemented and nonimplemented woreda in Gamo Gofa Zone, 2018

\begin{tabular}{|c|c|c|c|c|}
\hline Variables $s$ & Characteristic & $\begin{array}{c}\text { CLTSH-implemented (318) } \\
n(\%)\end{array}$ & $\begin{array}{c}\text { CLTSH nonimplemented (318) } \\
n(\%)\end{array}$ & $\begin{array}{c}\text { Total } \\
N(\%)\end{array}$ \\
\hline \multirow{2}{*}{ Latrine availability } & Yes & $240(75.5 \%)$ & $227(71.4 \%)$ & $467(73.4 \%)$ \\
\hline & No & $78(24.5 \%)$ & $91(28.6 \%)$ & $169(26.6 \%)$ \\
\hline \multirow{2}{*}{ Type of latrine } & VIP & $22(9.2 \%)$ & $35(15.4 \%)$ & $57(12.2 \%)$ \\
\hline & Traditional & $218(90.8 \%)$ & $192(84.6 \%)$ & $410(87.8 \%)$ \\
\hline \multirow{2}{*}{ Waste refuse disposal } & Proper & $384(83.3 \%)$ & $83(47.4 \%)$ & $467(73.4 \%)$ \\
\hline & Improper & $77(16.7 \%)$ & $92(52.6 \%)$ & $169(26.6 \%)$ \\
\hline \multirow{2}{*}{ Handwashing } & Yes & $111(46.2 \%)$ & $94(41.4 \%)$ & $205(43.9 \%)$ \\
\hline & No & $129(53.8 \%)$ & $133(58.6 \%)$ & $262(56.1 \%)$ \\
\hline \multirow{2}{*}{ Disposing infant feces } & In latrine & $276(86.8 \%)$ & $253(79.6 \%)$ & $529(83.2 \%)$ \\
\hline & Not in latrine & $42(13.2 \%)$ & $65(20.4 \%)$ & $107(16.8 \%)$ \\
\hline \multirow{2}{*}{ Source of drinking water } & Improved & $240(75.5 \%)$ & $212(66.7 \%)$ & $452(71.1 \%)$ \\
\hline & Unimproved & $78(24.5 \%)$ & $106(33.3 \%)$ & $184(28.9 \%)$ \\
\hline \multirow{2}{*}{ Time taken to obtain drinking water } & $<30 \mathrm{~min}$ & $259(81.4 \%)$ & $273(85.8 \%)$ & $532(83.6 \%)$ \\
\hline & $\geq 30 \mathrm{~min}$ & $59(18.6 \%)$ & $45(14.2 \%)$ & $104(16.4 \%)$ \\
\hline \multirow{2}{*}{ Per capita water consumption } & $<25$ liters & $237(74.5 \%)$ & $267(84 \%)$ & $504(79.2 \%)$ \\
\hline & $\geq 25$ liters & $81(25.5 \%)$ & $51(16 \%)$ & $132(20.8 \%)$ \\
\hline \multirow{4}{*}{ Water storage container } & Jerry can & $254(79.9 \%)$ & $175(55 \%)$ & 429 (67.5\%) \\
\hline & Plastic bucket & $17(5.3 \%)$ & $45(14.2 \%)$ & $62(9.8 \%)$ \\
\hline & Iron bucket & $5(1.6 \%)$ & $23(7.2 \%)$ & $28(4.4 \%)$ \\
\hline & Pot & $42(13.2 \%)$ & $75(23.6 \%)$ & $117(18.4 \%)$ \\
\hline \multirow{2}{*}{ Water storage cover } & Yes & $116(36.5 \%)$ & $78(24.5 \%)$ & $194(30.5 \%)$ \\
\hline & No & $202(63.5 \%)$ & $240(75.5 \%)$ & $442(69.5 \%)$ \\
\hline \multirow{2}{*}{ Water drawing from water storage } & By pouring & $35(42.5 \%)$ & $139(43.7 \%)$ & $274(43.1 \%)$ \\
\hline & By dipping & $183(57.5 \%)$ & $179(56.3 \%)$ & $362(56.9 \%)$ \\
\hline
\end{tabular}

TABLE 4: Behavior-related characteristics in selected CLTSH implemented and nonimplemented woreda in Gamo Gofa Zone, 2018.

\begin{tabular}{|c|c|c|c|c|}
\hline Variables & Characteristics & $\begin{array}{l}\text { CLTSH-implemented (318) } \\
n(\%)\end{array}$ & $\begin{array}{c}\text { CLTSH nonimplemented (318) } \\
n(\%)\end{array}$ & $\begin{array}{c}\text { Total } \\
N(\%)\end{array}$ \\
\hline \multirow{2}{*}{ Latrine utilization } & Properly & $132(55 \%)$ & $123(54.2 \%)$ & $255(54.6 \%)$ \\
\hline & Improperly & $108(45 \%)$ & $104(45.8 \%)$ & $212(45.4 \%)$ \\
\hline \multirow{2}{*}{ Washing hand with soap/ash } & Yes & $286(89.9 \%)$ & $224(70.4 \%)$ & $510(80.5 \%)$ \\
\hline & No & $32(10.1 \%)$ & $94(29.6 \%)$ & $126(19.5 \%)$ \\
\hline \multirow{2}{*}{ Critical handwashing times at least } & 3 (ref) & $280(88 \%)$ & $231(72.6 \%)$ & $511(80.3 \%)$ \\
\hline & $<3$ times & $38(12 \%)$ & $87(27.4 \%)$ & $125(19.7 \%)$ \\
\hline \multirow{3}{*}{ Utensils used to feed child } & Cup and spoon & $204(47.2 \%)$ & $230(53.5 \%)$ & $434(50.3 \%)$ \\
\hline & Bottle & $52(16.4 \%)$ & $49(15.4 \%)$ & $101(15.9 \%)$ \\
\hline & Hand & $62(19.5 \%)$ & $39(12.3 \%)$ & $101(15.9 \%)$ \\
\hline \multirow{2}{*}{ Water treatment } & Yes & $306(96.2 \%)$ & $309(97.2 \%)$ & $604(94.9 \%)$ \\
\hline & No & $12(3.8 \%)$ & $9(2.8 \%)$ & $32(5.1 \%)$ \\
\hline \multirow{3}{*}{ Times to complementary feeding started } & At 6 months & $161(50.6 \%)$ & $156(49.1 \%)$ & $317(49.8 \%)$ \\
\hline & Below 6 months & $90(29.3 \%)$ & $88(27.7 \%)$ & $178(28.0 \%)$ \\
\hline & Above 6 months & $67(21.1 \%)$ & $74(23.3 \%)$ & $141(22.2 \%)$ \\
\hline \multirow{2}{*}{ Rota vaccine } & Yes & $293(92.1 \%)$ & $261(82.1 \%)$ & $554(87.1 \%)$ \\
\hline & No & $25(7.9 \%)$ & $57(17.9 \%)$ & $82(12.9 \%)$ \\
\hline
\end{tabular}

and nonimplemented areas with $F$ value of 3.98 and $p$ value of 0.01 . The odds of developing diarrhea were 10 times higher among children whose families dispose refuses in open field when compared to whose family dispose wastes in pit (AOR; 9.70, 95\% CI (6.86-12.36)). There is difference in refuse disposal in open fields in CLTSH implemented and nonimplemented areas with $F$ value of 17.96 and $p$ value of 0.001 .
Regarding water shortage, holding other factors constant, children whose families use unimproved sources for drinking water were 6.5 times more likely (AOR; 6.58, 95\% CI $(2.82,10.39)$ ) to develop diarrhea when compared to children whose families used improved source of drinking water. There is a difference in using unimproved water as drinking water in CLTSH implemented and nonimplemented areas with $F$ value of 3.52 and $p$ value of 0.02 . 


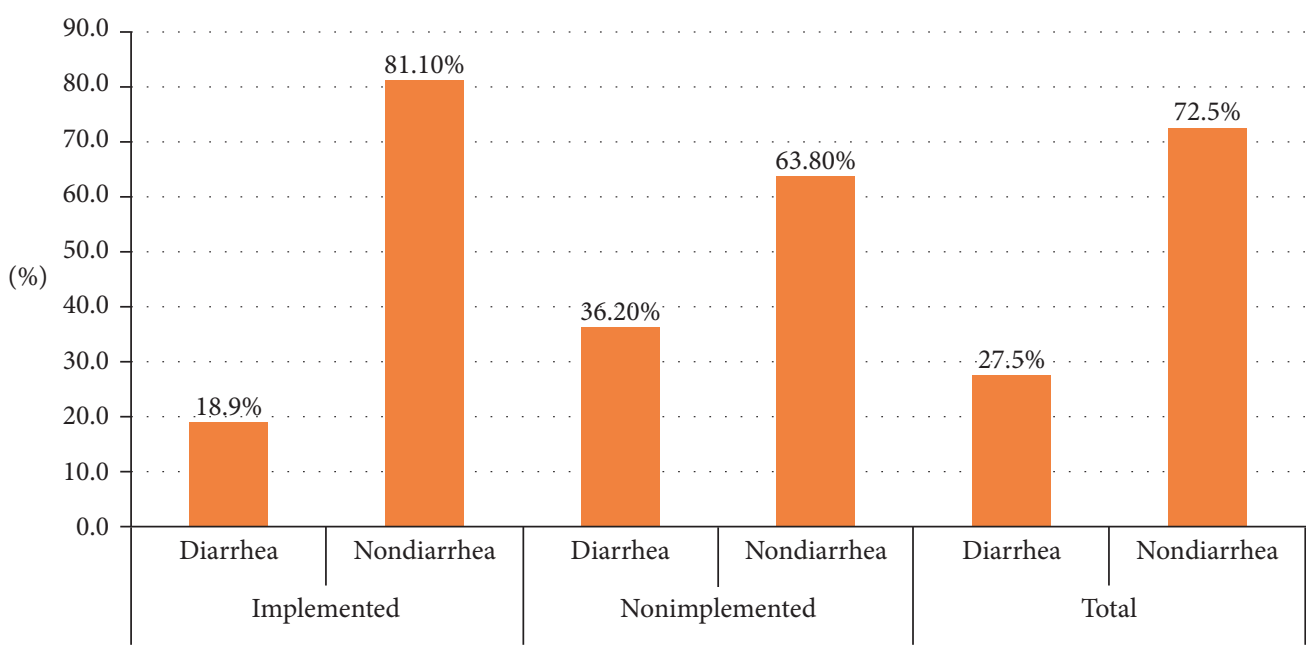

FIGURE 3: Bar chart shows the prevalence of diarrhea among under-five in CLTSH implemented and nonimplemented areas in Gamo Gofa Zone, 2018.

TABLE 5: Bivariate analysis of sociodemographic variables related to diarrhea among under-five in selected CLTSH implemented and nonimplemented woredas in Gamo Gofa Zone, March 2018.

\begin{tabular}{|c|c|c|c|c|c|}
\hline \multirow{2}{*}{ Variables } & \multirow{2}{*}{ Characteristics } & \multicolumn{2}{|c|}{ Diarrhea status } & \multirow{2}{*}{ COR $[95 \% \mathrm{CI}]$} & \multirow{2}{*}{$p$ value } \\
\hline & & CLTSH, $n(\%)$ & Non-CLTSH, $n(\%)$ & & \\
\hline \multirow{2}{*}{ Caretaker of index child } & Mother (ref) & $52(86.7 \%)$ & $66(57.4 \%)$ & 1.00 & \\
\hline & Caretaker & $107(61.1 \%)$ & $68(38.9 \%)$ & $4.89[3.22,7.43]$ & 0.001 \\
\hline \multirow{3}{*}{ Age of caretaker } & $\geq 35$ years & $22(36.7 \%)$ & $24(20.9 \%)$ & 1.00 & \\
\hline & $25-34$ years & $15(25.0 \%)$ & $56(48.7 \%)$ & $0.92[0.59,1.42]$ & 0.7 \\
\hline & $15-24$ years & $23(38.3 \%)$ & $35(30.4 \%)$ & $1.25[0.79,1.98]$ & 0.34 \\
\hline \multirow{5}{*}{ Marital status } & Married (ref) & $44(73.3 \%)$ & $61(53.0 \%)$ & 1.00 & \\
\hline & Single & $3(5.0 \%)$ & $16(13.9 \%)$ & $1.47[0.81,2.64]$ & 0.2 \\
\hline & Widowed & $8(13.3 \%)$ & $15(13.0 \%)$ & $2.22[1.24,3.96]$ & 0.01 \\
\hline & Divorced & $3(5.0 \%)$ & $16(13.9 \%)$ & $1.36[0.76,2.44]$ & 0.3 \\
\hline & Separated & $2(3.3 \%)$ & $7(6.10 \%)$ & $1.26[0.56,2.83]$ & 0.57 \\
\hline \multirow{3}{*}{ Monthly income } & $<1000$ ETB & $44(73.3 \%)$ & $72(62.6 \%)$ & $0.88[0.58,1.33]$ & 0.53 \\
\hline & 1000-2000 ЕTВ & $12(20.0 \%)$ & $39(33.9 \%)$ & $1.29[0.76,2.21]$ & 0.34 \\
\hline & $\geq 2000$ ETB & $4(6.7 \%)$ & $4(3.50 \%)$ & 1.00 & \\
\hline \multirow{2}{*}{ Number of $<5$ children } & 1 & $52(86.7 \%)$ & $100(87.0 \%)$ & 1.00 & \\
\hline & $\geq 2$ & $8(13.3 \%)$ & $15(13.0 \%)$ & $0.99[0.59,1.66]$ & 0.98 \\
\hline \multirow{4}{*}{ Age of index child } & $<6$ months & $14(23.3 \%)$ & $25(21.7 \%)$ & 1.00 & \\
\hline & $6-11$ months & $15(25.0 \%)$ & $35(30.4 \%)$ & $2.64[1.63,4.29]$ & 0.001 \\
\hline & $12-23$ months & $15(25.0 \%)$ & $16(13.9 \%)$ & $1.89[1.11,3.23]$ & 0.001 \\
\hline & $\geq 24$ months & $16(26.7 \%)$ & $39(33.9 \%)$ & $3.75[2.30,6.11]$ & 0.001 \\
\hline \multirow{2}{*}{ Family size of household } & $<5$ & $40(66.7 \%)$ & $64(55.7 \%)$ & 1.00 & \\
\hline & $\geq 5$ & $20(33.3 \%)$ & $51(44.3 \%)$ & $0.93[0.65,1.33]$ & 0.69 \\
\hline
\end{tabular}

Regarding behavioral factors, children whose mothers used only water to wash their hands were about 6 times more likely $(\mathrm{AOR}=5.89 ; 95 \% \mathrm{CI}:(3.56-10.59))$ to have diarrhea than children whose mothers used water with soap or ash to wash their hands. There is difference in only using water during handwashing in CLTSH implemented and nonimplemented areas with $F$ value of 13.79 and $p$ value of 0.001 . Children started complementary feeding above six months were 5 (AOR: 5.21, 95\% CI (2.20-8.05)) and below six months were 6 (AOR: 5.62, 95\% CI (3.49-9.6)) times more likely to have child diarrhea compared to children started supplementary feeding at six months. There is no difference in time to complementary feeding started in CLTSH implemented and nonimplemented areas with $F$ value of 0.22 and $p$ value of 0.80 . Children whose caretakers use bottle to feed their child were 4 times (AOR: $3.88,95 \%$ CI (1.78-8.46)) more likely to have child diarrhea compared to children whose caretakers use cup and spoon for feeding. There is no difference in utensils used to feed child in CLTSH implemented and nonimplemented areas with $F$ value of 0.339 and $p$ value of 0.79 (see Table 8 ). 
TAвLE 6: Bivariate analysis of environmental factors related to diarrhea among under-five in selected CLTSH implemented and nonimplemented woredas in Gamo Gofa Zone, March 2018.

\begin{tabular}{|c|c|c|c|c|c|}
\hline \multirow{2}{*}{ Variables } & \multirow{2}{*}{ Characteristics } & \multicolumn{2}{|c|}{ Diarrhea status } & \multirow{2}{*}{ COR $[95 \% \mathrm{CI}]$} & \multirow{2}{*}{$p$ value } \\
\hline & & CLTSH, $n(\%)$ & Non-CLTSH, $n(\%)$ & & \\
\hline \multirow{2}{*}{ Latrine availability } & Yes & $34(56.7 \%)$ & $69(60.0 \%)$ & 1.00 & \\
\hline & No & $26(43.3 \%)$ & $46(40.0 \%)$ & $2.62[1.80,3.82]$ & 0.001 \\
\hline \multirow{2}{*}{ Refuse disposal } & Proper & $44(73.3 \%)$ & $39(33.9 \%)$ & 1.00 & \\
\hline & Improper & $16(26.7 \%)$ & $76(66.1 \%)$ & $5.53[3.76,8.12]$ & 0.001 \\
\hline \multirow{2}{*}{ Handwashing } & Yes & $6(17.6 \%)$ & $19(27.5 \%)$ & 1.00 & \\
\hline & No & $28(82.4 \%)$ & $50(72.5 \%)$ & $3.05[1.86,5.01]$ & 0.001 \\
\hline \multirow{2}{*}{ Disposing infant feces } & Proper & $54(90.0 \%)$ & $88(76.5 \%)$ & 1.00 & \\
\hline & Improper & $6(10.0 \%)$ & $27(23.5 \%)$ & $1.21[0.77,1.91]$ & 0.4 \\
\hline \multirow{2}{*}{ Source of drinking water } & Improved & $31(51.7 \%)$ & $42(36.5 \%)$ & 1.00 & \\
\hline & Unimproved & $29(48.3 \%)$ & $73(63.5 \%)$ & $6.46[4.4,9.48]$ & 0.001 \\
\hline \multirow{2}{*}{ Time taken to obtain drinking water } & $<30 \mathrm{~min}$ & $46(76.7 \%)$ & $96(83.5 \%)$ & 1.00 & \\
\hline & $\geq 30 \mathrm{~min}$ & $14(23.3 \%)$ & $19(16.5 \%)$ & $1.28[0.81,2.01]$ & 0.293 \\
\hline \multirow{2}{*}{ Per capita water consumption per day } & $<25$ litres & $39(65 \%)$ & $102(88.7 \%)$ & 1.00 & \\
\hline & $\geq 25$ litres & $21(35 \%)$ & $13(11.3 \%)$ & $0.72[0.46,1.10]$ & 0.126 \\
\hline
\end{tabular}

TABLE 7: Bivariate analysis of behavioral factors related to diarrhea among under-five in selected CLTSH implemented and nonimplemented woredas in Gamo Gofa Zone, March 2018.

\begin{tabular}{|c|c|c|c|c|c|}
\hline \multirow{2}{*}{ Variables } & \multirow{2}{*}{ Characteristics } & \multicolumn{2}{|c|}{ Diarrhea status } & \multirow{2}{*}{ COR } & \multirow{2}{*}{$p$ value } \\
\hline & & CLTSH, $n(\%)$ & Non-CLTSH, $n(\%)$ & & \\
\hline \multirow{2}{*}{ Latrine utilization } & Proper & $19(55.9 \%)$ & $35(50.7 \%)$ & 1.00 & \\
\hline & Improper & $15(44.1 \%)$ & $34(49.3 \%)$ & $1.12[0.72,1.74]$ & 0.61 \\
\hline \multirow{2}{*}{ Washing hand with soap/ash } & Yes (ref) & $26(76.5 \%)$ & $29(25.2 \%)$ & 1.00 & \\
\hline & No & $8(23.5 \%)$ & $86(74.8 \%)$ & $5.17[3.06,8.74]$ & 0.001 \\
\hline \multirow{3}{*}{ Utensils used to feed child } & Cup and spoon & $25(41.7 \%)$ & $62(53.9 \%)$ & 1.00 & \\
\hline & Bottle & $17(28.3 \%)$ & $30(26.1 \%)$ & $2.89[1.85,4.52]$ & 0.001 \\
\hline & Hand & $18(30.0 \%)$ & $23(20.0 \%)$ & $2.34[1.48,3.68]$ & 0.001 \\
\hline \multirow{2}{*}{ Critical handwashing times at least } & 3 (ref) & $29(85.3 \%)$ & $50(72.5 \%)$ & 1.00 & \\
\hline & $<3$ & $5(14.7 \%)$ & $19(27.5 \%)$ & $1.48[0.87,2.52]$ & 0.15 \\
\hline \multirow{2}{*}{ Water treatment } & Yes (ref) & $57(95.0 \%)$ & $111(96.5 \%)$ & 1.00 & \\
\hline & No & $3(5.0 \%)$ & $4(3.5 \%)$ & $1.43[0.56,3.65]$ & 0.45 \\
\hline \multirow{4}{*}{ Water storage container } & Jerry can (ref) & $49(81.7 \%)$ & $32(27.8 \%)$ & 1.00 & \\
\hline & Plastic bucket & $5(8.3 \%)$ & $26(22.6 \%)$ & $4.29[2.47,7.47]$ & 0.001 \\
\hline & Iron bucket & $6(1.7 \%)$ & $57(10.4 \%)$ & $3.72[1.71,8.13]$ & 0.001 \\
\hline & Pot & $5(8.3 \%)$ & $45(39.1 \%)$ & $3.21[2.1,4.97]$ & 0.001 \\
\hline \multirow{2}{*}{ Water storage cover } & Yes & $21(35.0 \%)$ & $29(25.2 \%)$ & 1.00 & \\
\hline & No & $39(65.0 \%)$ & $86(74.8 \%)$ & $1.14[0.78 .1 .67]$ & 0.52 \\
\hline \multirow{2}{*}{ Methods water drawing } & By pouring & $26(43.3 \%)$ & $54(47.0 \%)$ & 1.00 & \\
\hline & By dipping & $34(56.7 \%)$ & $61(53.0 \%)$ & $0.86[0.61,1.23]$ & 0.41 \\
\hline \multirow{3}{*}{ Times to complementary feeding started } & At 6 months & $18(30.0 \%)$ & $28(24.3 \%)$ & 1.00 & \\
\hline & Below 6 months & $25(41.7 \%)$ & $46(40.0 \%)$ & $3.91[2.54,6.03]$ & 0.001 \\
\hline & Above 6 months & $17(28.3 \%)$ & $41(35.7 \%)$ & $4.12[2.6,6.5]$ & 0.001 \\
\hline \multirow{2}{*}{ Rota vaccine } & Yes (ref) & $47(78.3 \%)$ & $71(61.7 \%)$ & 1.00 & \\
\hline & No & $13(21.7 \%)$ & $44(38.3 \%)$ & $5.54[3.49,8.78]$ & 0.001 \\
\hline
\end{tabular}

\section{Discussion}

The finding of this study revealed the prevalence and associated factors of diarrhea. The overall diarrhea prevalence was $27.5 \%$ which was $18.9 \%$ in implemented and $36.2 \%$ in nonimplemented woredas and the result has a significant difference in the occurrence of diarrhea. The associated factors of acute diarrhea in this study were age of index child, availability of handwashing facilities, mothers using only water to wash their hands, open pit waste disposal, source of 
TABLE 8: Multivariable analyses of factors related to diarrhea among under-five in selected CLTSH implemented and nonimplemented woredas in Gamo Gofa Zone, March 2018.

\begin{tabular}{|c|c|c|c|c|}
\hline \multirow{2}{*}{ Characteristics } & \multicolumn{2}{|c|}{ Diarrhea status } & \multirow{2}{*}{ COR $[95 \% \mathrm{CI}]$} & \multirow{2}{*}{ AOR $[95 \% \mathrm{CI}]$} \\
\hline & CLTSH, $n(\%)$ & Non-CLTSH, $n(\%)$ & & \\
\hline \multicolumn{5}{|l|}{ Age of index child } \\
\hline$<6$ months & $14(23.3 \%)$ & $25(21.7 \%)$ & 1.00 & 1.00 \\
\hline 6-11 months & $15(25.0 \%)$ & $16(13.9 \%)$ & $1.89[1.11,3.2]$ & $1.38[0.49,3.84]$ \\
\hline $12-23$ months & $15(25.0 \%)$ & $35(30.4 \%)$ & $2.64[1.6,4.29]$ & $1.58[1.5,8.56]^{*}$ \\
\hline$\geq 24$ months & $16(26.7 \%)$ & $39(33.9 \%)$ & $3.75[2.3,6.11]$ & $5.23[2.12,12.8]^{*}$ \\
\hline \multicolumn{5}{|c|}{ Handwashing facilities } \\
\hline Yes & $6(17.6 \%)$ & $19(27.5 \%)$ & 1.00 & 1.00 \\
\hline No & $28(82.4 \%)$ & $50(72.5 \%)$ & $3.05[1.86,5.0]$ & $4.03[1.93,8.42]^{*}$ \\
\hline \multicolumn{5}{|c|}{ Handwashing with soap/ash } \\
\hline Yes & $26(76.5 \%)$ & $29(25.2 \%)$ & 1.00 & 1.00 \\
\hline No & $8(23.5 \%)$ & $86(74.8 \%)$ & $5.17[3.06,8.74]$ & $5.89[3.56,10.59]^{*}$ \\
\hline \multicolumn{5}{|l|}{ Refuse disposal } \\
\hline Proper & $44(73.3 \%)$ & $39(33.9 \%)$ & 1.00 & 1.00 \\
\hline Improper & $16(26.7 \%)$ & $76(66.1 \%)$ & $5.53[3.76,8.12]$ & $5.9[2.61,9.78]^{*}$ \\
\hline \multicolumn{5}{|c|}{ Source of drinking water } \\
\hline Improved & $31(51.7 \%)$ & $42(36.5 \%)$ & 1.00 & 1.00 \\
\hline Unimproved & $29(48.3 \%)$ & $73(63.5 \%)$ & $6.46[4.4,9.48]$ & $6.58[2.82,10.39]^{*}$ \\
\hline \multicolumn{5}{|c|}{ Times to complementary feeding started } \\
\hline At 6 months & $18(30.0 \%)$ & $28(24.3 \%)$ & 1.00 & 1.00 \\
\hline Below 6 months & $25(41.7 \%)$ & $46(40.0 \%)$ & $3.9[2.54,6.03]$ & $5.62[3.49,9.6]^{*}$ \\
\hline Above 6 months & $17(28.3 \%)$ & $41(35.7 \%)$ & $4.12[2.6,6.51]$ & $5.21[2.2,8.05]^{*}$ \\
\hline \multicolumn{5}{|c|}{ Utensils used to feed child } \\
\hline Cup and spoon & $25(41.7 \%)$ & $62(53.9 \%)$ & 1.00 & 1.00 \\
\hline Bottle & $17(28.3 \%)$ & $30(26.1 \%)$ & $2.89[1.85,4.52]$ & $3.88[1.78,8.46]^{*}$ \\
\hline Hand & $18(30.0 \%)$ & $23(20.0 \%)$ & $2.34[1.48,3.68]$ & $2.04[0.82,5.02]$ \\
\hline
\end{tabular}

drinking water, times that children started supplementary feeding, and utensils used to feed child. From these factors, source of drinking water, refuse disposal, and only water during handwashing were significantly different between implemented and nonimplemented.

The prevalence of diarrhea among implemented woredas was $18.9 \%$ which is much higher as compared with CLTSH kebeles diarrhea prevalence in India that is 2.72\% [17] and the prevalence in Dangla woreda Amhara region is $9.9 \%$ [8]. However, it is similar to the study done in Kersa District, Jimma Zone (18.9\%). The reason behind the increment of diarrhea prevalence in the study area may be due to the quality of CLTSH implementation, declaration, certification, and follow-up after intervention. The prevalence of under-five diarrhea in nonimplemented woredas was $36.2 \%$ which is consistent with Dangla woreda (36.1\%) [8] and national under-five diarrhea prevalence that ranges from $11 \%$ to $38 \%$ [18] and in contrarily higher than Jimma zone Kersa woreda $22.22 \%$ [9] and India $15.9 \%$ [17]. This difference may come because of the people's way of life, waste refuse disposal, and handwashing practice. The prevalence of under-five diarrhea among CLTSH and non-CLTSH woredas has significant difference with $X^{2}=23.3$ and $p=0.001$. It was similar to the study done in Dangla woreda which has significant difference. However, the study done in Jimma zone showed that there is no significant difference between the two communities.
In this particular study, there was a significant association between the starting times of supplementary feeding. The times that children started complementary feeding above six months and below six months were more likely to have child diarrhea compared to children started at six months. This result was similar to the study done in Kotebe [19]. This is might be due to contaminated feeding, no maturity of the gastrointestinal system, decreased intake of breastfeeding in early and not enough feeding, and slow adoption of feeding after six months.

Children whose age was greater than 6 months were highly associated with under-five diarrhea when compared with children whose age was less than six months. This was in line with the study done in Sidama Zone and Farta woreda, Northern Ethiopia [16, 20]. This might be the introduction of contaminated weaning foods and handwashing practice of caretaker method of children feeding.

Households with no handwashing facilities were more likly to develop diarrhea compared with the children household having handwashing facilities. This result is consistent with the study done in Kersa District, Jima Zone, Farta woreda, Northern Ethiopia [9, 12, 20]. Presence may increase the utilization of handwashing rather and this may contribute to the reduction in the risk. This has an implication that the presence of handwashing facility does have a great contribution to the prevention of under-five diarrhea. This result might be because of the less follow-up of CLTSH implementation and less utilization of handwashing. 
Households that did not use soap/ash for handwashing were more likely to develop diarrhea compared to children whose caretakers use soap/ash during handwashing times. This result was higher compared to a study conducted in Jabithennan District, Northern Ethiopia, Farta woreda, Northern Ethiopia, and Kersa District, Jimma Zone $[9,20,21]$. This might be due to information about critical handwashing times, less practice in local materials used to wash hands, and less information in nonimplemented area. Since soaps and ash are antimicrobial agents, it has the power to prevent diarrhea and occurrence of other hygienerelated diseases.

Another factor associated with this study was refuse disposal, and open disposal increases the chance of diarrhea occurrence compared to pit disposal. These results were higher compared to a study done in Kersa District, Jimma Zone [9]. The increment might be because of the differences in the implementation status of CLTSH and health extension program, living conditions of communities, and agroecological differences.

Regarding the access to drinking water, children whose caretakers use unprotected source of drinking water and river water had a higher chance of diarrhea occurrence as compared to those using pipe as a source of drinking water. This result was higher compared to a study done in Derashe woreda [22]. This difference might be due to difference in investment on water sources, communities handling of installed improved water sources, mixed use of water from both protected and unprotected sources, contamination during transport, and storage or lack of homogeneity in source of water.

And also the odds of diarrheal occurrence in under-five children were much higher among children who used feeding utensils hand and bottle methods compared to cup and spoon methods of feeding. This finding was in accordance with other studies in Jabithennan District [21]. This finding might be associated with improper cleaning of bottles.

\section{Strengths and Limitations of the Study}

\subsection{Strength of the Study}

(i) The analytic approach and community-based study help to detect the true magnitude and factors and help to generalize the finding.

\subsection{Limitation of the Study}

(i) Recall bias may occur on two-week occurrence of diarrhea and it may underestimate the magnitude. This was minimized using reported incident cases within two-week period

\section{Conclusion and Recommendation}

6.1. Conclusion. There is difference in the occurrence of diarrhea, refuse disposal, source of drinking water, and washing hands with soap/ash in CLTSH implemented and nonimplemented areas. The magnitude of diarrhea among under-five children in CLTSH implemented and in nonimplemented areas was $18.9 \%$ and $36.2 \%$ and the prevalence was high in nonimplemented woredas. Generally, the majority of possible factors associated with the occurrence of under-five childhood diarrhea in this study were age of child, handwashing facilities, refuse disposal, source of drinking water, washing hands with soap/ash, supplementary feeding commencement time, and utensils used to feed child.

6.2. Recommendations. The following recommendations are forwarded based on the findings of the study:

To district or zonal health department

(i) Providing continuous and regular supervision/information to the community focusing on environmental hygiene, handling source of water, and children feeding

To woreda health office

(i) The district health office should do better to improve services, i.e., handwashing facilities, source of drinking water, solid waste disposal practices, and children feeding that are directly linked with under-five diarrhea

(ii) Also it is better to construct improved water source so as to provide safe water in the community

To community

(i) The community should improve handwashing practice. This could be achieved by using simple home-based methods like ash.

(ii) Overall hygienic practices during child supplementary feeding commencement should get emphasis so as to control and prevent under-five childhood diarrhea morbidity.

To researchers

(i) The researchers are better to do further investigation on the impact and role of being CLTSH implementation as comprehensive package on diarrhea occurrences and factors associated with diarrhea occurrence.

\section{Data Availability}

The data used to support the findings of this study are available from the corresponding author upon request.

\section{Ethical Approval}

Ethical approval and clearance were obtained from the Ethical Review Committee of Arba Minch University, College of Medicine and Health Sciences. Following the approval, an official letter of cooperation was written to Gamo Gofa Zone Health Department by the Department of Public Health of Arba Minch University. Explanation on the objective of the research was provided to the concerned personnel at zonal level. Support letter that was submitted to the Woreda Health Office was obtained from the Zonal 
Health Department. Similarly, the letter was written to the health facilities and kebeles for cooperation.

\section{Consent}

Informed oral consent was obtained from the respondents after explaining the purpose of the study. Participants were assured of confidentiality with regard to all information acquired. In addition, withdrawal from the study during the interview has been guaranteed to all the study participants at any time. Finally, data were collected after assuring the confidentiality nature of responses and obtaining oral consent from the study participants.

\section{Conflicts of Interest}

The authors declare that they have no conflicts of interest.

\section{Authors' Contributions}

AA contributed to proposal development, pretesting of the questionnaires, data cleaning, data analysis, and manuscript preparation. WG provided advice during proposal development and was involved in pretesting the questionnaire and supervising the data collectors and data entry. GG provided advice during proposal development and was involved in pretesting the questionnaire and supervising the data collectors and data entry. All authors have read and approved the final manuscript.

\section{Acknowledgments}

The authors would like to thank Arba Minch University, College of Medical Sciences. They are also grateful to the cooperation of the study participants and to Gamo Gofa Zone Health Department, Selected Woreda Health Office, for their kind assistance when preparing the proposal and in the final data collection.

\section{References}

[1] G. Frank, Adoption of Community-Led Total Sanitation Guidance for Programming of CLTS in Tear Fund-Supported Projected, 2014.

[2] L. Mehta, Community-led Total Sanitation (CLTS) across the Seas. Experiences from Africa with a Special Emphasis on Ethiopia, Ripple, San Francisco, CA, USA, 2016.

[3] A. Dunc, L. Jon, S. Beth, and T. David, "Sanitation and health," PLoS Medicine, vol. 7, no. 11, 2013.

[4] F-MOH/UNICEF/WSSCC-GSE, Out Come Evaluation of Community-Led Total Sanitation and Hygiene, 2015.

[5] Nelson, Text Book of Peadiatric, 7th edition, 2006.

[6] UN-Water, Challenge of Extending and Sustaining Services Global Analysis and Assessment of Sanitation and Drinking Water GLAAS Report, UN-Water, Geneva, Switzerland, 2012.

[7] UNICEF, Water Sanitation and Hygiene Report, UNICEF, New York, NY, USA, 2014.

[8] M. Abireham, "Assessment of diarrhea in under-five children: a comparative cross-sectional study in open defecation and open defecation free rural settings in Dangla District, North West Ethiopia: Addis Ababa," Journal of Environmental and Public Health, vol. 2018, Article ID 4271915, 8 pages.
[9] E. Negasa, B. Abebe, and T. Gudina, "Implementation of community-led total sanitation and hygiene approach on the prevention of diarrheal disease in Kersa district, Jimma zone Ethiopia," Science Journal of Public Health, vol. 3, no. 5, pp. 669-676, 2015.

[10] The Burden of Diarrheal Diseases Among Children under Five Years of Age in Arba Minch District, Southern Ethiopia, and Associated Risk Factors: A Cross-Sectional Study, 2014.

[11] WHO, The Global Burden of Disease: 2014 Update, World Health Organization, Geneva, Switzerland, 2014.

[12] M. Dessalegn, A. Kumie, and W. Tefera, "Predictors of underfive childhood diarrhea: mecha district, west Gojam, Ethiopia," The Ethiopian Journal of Health Development, vol. 25, pp. 194-196, 2011.

[13] WHO and UNICEF, Types of Improved Drinking-Water Source on the JMP Website, WHO, UNICEF, New York, NY, USA, 2012.

[14] WHO and UNICEF, Improved and Unimproved Water and Sanitation Facilities, WHO, New York, NY, USA, 2015.

[15] S. Cavill, R. Chambers, and N. Vernon, "Sustainability and CLTS. Taking stock," Frontiers of CLTS, vol. 2, no. 4, p. 18, 2015.

[16] T. Bilkutkenaw, "Predictors of child diarrheal diseases in cltsh and phast approach implemented areas in Shebedino District, Sidama Zone, South Ethiopia," MS thesis, Addis Ababa University, Addis Ababa, Ethiopia, 2013.

[17] C. Tapas, G. Sam, J. Bhatt, P. Rao, P. Meshram, and K. Singh, "Cross-sectional health indicator study of open defecationfree villages in Madhya Pradesh, India," South Asian Sanitation, vol. 27, no. 3, 2008.

[18] A. Andualem and K. Abera, "Assessment of the impact of latrine utilization on diarrhoeal diseases in the rural community of hulet Ejju Enessie woreda, East Gojjam zone, Amhara region," The Ethiopian Journal of Health Development, vol. 24, no. 2, 2010.

[19] T. Aklilu and A. Zewdie, "Determinants of under-five childhood diarrhea in Kotebe health center, Yeka sub city, Addis Ababa, Ethiopia: a case control study," Global Journal of Medical Research, vol. 14, 2014.

[20] G. Genet, K. Abera, and H. Desta, "Magnitude and associated factors of diarrhea among under five children in Farta Wereda, North west Ethiopia," Quality in Primary Care, vol. 25, no. 4, pp. 199-207, 2017.

[21] A. Zelalem, A. Kassawmar, and T. Molalign, "Prevalence and determinants of acute diarrhea among children younger than five years old in Jabithennan District, Northwest Ethiopia," BMC Public Health, vol. 17, 2014.

[22] G. Wanzahun and M. Bezatu, "Determinants of acute diarrhoea among children under five years of age in Derashe District," Rural and Remote Health, vol. 13, p. 2329, 2013. 